\title{
Codes and coding processes in pigeon short-term memory
}

\author{
H. L. ROITBLAT \\ Columbia University, New York, New York 10027
}

\begin{abstract}
The delayed matching-to-sample (DMTS) task was used in three experiments to investigate how pigeons code information about sample stimuli. In all experiments, each trial consisted of a signaled presentation of a sample stimulus for a fixed duration followed, after some delay, by the presentation of three comparison stimuli. After incorrect first choices, the bird was allowed a second choice between the romaining two stimuli. It was found, in Experiment 1, that the probability of a second choice error declined with increasing sample duration. This result is consistent with a gradual short-term memory encoding process but not with a simple two-state all-or-none process. In the second experiment, it was found that the distribution of first-choice errors was affected by the particular sample occurring on a trial. This result is inconsistent with a two-stage discrete state memory/attention model based on the assumption that encoding of the sample and attention to the comparison stimuli are both independent all-or-none processes. The third experiment involved symbolic DMTS, in which the sample stimuli varied along a dimension different from that along which the comparison stimuli varied. With increasing delay between sample and comparison stimulus presentations, the pigeons were more likely to confuse test stimuli than to confuse sample stimuli. The results of these experiments lead to the conclusion that pigeon DMTS performance depends on a gradual encoding process in which a representation isomorphic with the test stimuli is generated and maintained.
\end{abstract}

The delayed matching-to-sample (DMTS) task is ideally suited to the study of short-term memory in nonverbal animals. DMTS involves the presentation of a sample stimulus, followed by a delay, followed by presentation of two or more comparison or test stimuli. Responses to the comparison stimulus that matches the sample stimulus are rewarded. Because the sample stimulus is not present when the comparison stimuli are presented, accurate performance despite delays ranging up to several seconds implies that the subject has access, when tested, to a maintained representation (memory) of the information contained in the sample.

In one of the earliest systematic analyses of shortterm memory in pigeons, Roberts (1972) found that performance tended to improve with increasing sample duration. "The most elementary theory which one might advance to account for these findings holds

This research was presented in partial fulfillment of the requirements for a PhD degree from the University of California, Berkeley. This research was supported by National Institute of Mental Health Grant MH 22153 to Donald A. Riley and by National Institute of Medical Sciences Grant GM 01207-10sl to The Institute of Human Learning, University of California, Berkeley. I thank Donald Riley for his lasting support and Thomas Wickens for help in developing the models. Reprint requests should be sent to $H$. L. Roitblat, Department of Psychology, 406 Schermerhorn Hall, Columbia University, New York, New York 10027. that each presentation of the sample stimulus"' (operationally defined as a peck to it) "adds an increment to the strength of a unitary memory trace, ... and that the probability of a correct choice is directly related to trace strength" (Roberts, 1972, p. 76). Trace strength is assumed to affect the probability of a correct choice via the degree of match between the trace of the sample and the correct comparison stimulus. "A subject compares the stimuli presented on the side keys (the comparison stimuli) with a trace of the sample stimulus stored in memory. If a match between trace and test stimulus is made, a correct choice results" (Roberts \& Grant, 1976, p. 88).

There are two obvious ways in which trace strength could increase over time. First, since the usual measure of performance on a DMTS task is the percentage of correct responses, it is possible that trace strength could increase in an all-or-none fashion with some probability during each small instant of sample presentation. All-or-none models have been used quite successfully in conditioning (Pfautz \& Wagner, 1976; Theios \& Brelsford, 1966; Wagner, 1976) and in the acquisition of matching-to-sample (Rodewald, 1974), and they have proved powerful in dealing with human paired-associate memory (e.g., Bower, 1961; Rock, 1957). Performance then depends on the probability of having encoded the sample information before the end of the sample presentation. Correct choices could result either from having already en- 
coded or from a correct guess and would increase with increasing sample duration.

The obvious alternative to a discrete-state, unitarymemory process is one in which the memory representation forms gradually. According to one model of this type, the pigeon generates a visual code that is "veridical or at least isomorphic" (Farthing, Wagner, Gilmour, \& Waxman, 1977, p. 528) with the sample stimulus. At the same time that the visual code is developing, there is a second code generated, unique to each particular sample stimulus, which may contain more abstracted information about the sample. The pigeon is capable of maintaining this abstract code in memory through active rehearsal. Farthing et al. (1977) also assume that the formation of both codes takes time, that both may occur simultaneously, and that since the abstracted code depends on a higher level of processing, it probably takes more time to develop than does the veridical code. Thus, with increasing sample duration, the representations become more fully developed, increasing the likelihood of a correct match (cf. Riley \& Roitblat, 1978; Roitblat, Greene, \& Riley, Note 1).

These two models represent extremes on a continuum. There are probably a large number of alternative models in which the coding process is either intermediate between these extremes of all-or-none vs. pure strength changes or is a combination of the two. As extremes, however, they represent relatively "pure" cases. A first experimental assault on the nature of the coding process would probably benefit by restricting itself to these two relatively simple cases. [See Roitblat(1978) for a more complete development of these and a third model; also see Wickens and Greitzer (1975) for the development of similar models applied to human paired associate learning.]

According to the all-or-none model, the pigeon's short-term memory system is assumed to be in one of two possible memory states following the onset of the sample stimulus: At the beginning of a trial, the memory system is assumed to start in state $\mathrm{CO}$, a pure guessing state in which the subject has no information about the identity of the sample stimulus. At some point during sample presentation, the subject may pass from state $\mathrm{C} 0$ to the second memory state, $\mathrm{Cl}$, in which the subject has all the available information and choices are always correct. State transitions correspond to a Poisson process in which the probability of changing state is constant for all brief intervals and independent of the time since the onset of the sample stimulus. Once encoded into state $\mathrm{Cl}$, it is assumed that no more state changes will occur before the end of the trial. The essential feature of the all-or-none model is the assumption of stationarity, that all errors result from state $\mathrm{CO}$ and that the subject is in the same state and has the same probability of changing from state $\mathrm{C} 0$ after the first millisecond of sample duration as after the 10th, or the 100th.

The "gradual" model is based on the assumption that there are levels of memory strength intermediate between a perfect encoding and a complete lack of information. Similar models have been proposed by Bush and Mosteller (1955), Estes (1955), Mackintosh (1975), Neimark and Estes (1967), and Rescorla and Wagner (1972) to account for trial-to-trial performance. According to the gradual model, performance depends on the accrued strength of the sample stimulus representation. Both the all-or-none and the gradual models predict an exponential decline in the probability of an error with increasing sample duration.

The main feature of the simple all-or-none model that allows it to be discriminated from other models is the single source of errors. The occurrence of an error is therefore prima facie evidence that no encoding has yet occurred.

In order to exploit this stationary feature of the all-or-none model, the matching-to-sample task was modified so that three comparison stimuli were available on every trial. By this modification, it was possible to allow second choices on those trials on which the first choice was an error. Since the opportunity to make a second choice was contingent on a firstchoice error, it must, according to the all-or-none model, also be contingent on being in state $\mathrm{C} 0$ and so be a guess. The all-or-none model predicts random second choices at all sample durations and all levels of first-choice accuracy.

The gradual model, on the other hand, assumes that a first-choice error can occur even when the subject has some information regarding the identity of the sample. Following a first-choice error, the subject scans memory a second time, at which point the number of alternatives is reduced from three to two and there is once again a probability that any given unit of trace strength will contribute to a match. Therefore, the gradual model predicts that second choices will contain information about the sample and that secondchoice performance will also improve with increases in sample duration.

\section{EXPERIMENT 1}

The first experiment compared the predictions of the all-or-none and the gradual models using a DMTS procedure with three alternative choice stimuli available on each trial.

\footnotetext{
Method

Subjects. Five experimentally naive Silver King pigeons served as subjects. The birds were maintained at between $75 \%$ and $80 \%$ of their free-feeding weights. All birds were at least 1 year old at the beginning of the experiment. Birds 17,49 , and 71 were trained with color stimuli; Birds 60 and 61 were trained with form stimuli.

Apparatus. Experimental sessions were conducted in standard three-key operant chambers. The front wall of these chambers held
} 
three translucent pecking keys $(2.4 \mathrm{~cm}$ in diameter) arranged in a line $5.8 \mathrm{~cm}$ apart and $23.3 \mathrm{~cm}$ above the wire-mesh floor. A food hopper $(5.5 \times 5.2 \mathrm{~cm}$ high) containing mixed grain was located $9.2 \mathrm{~cm}$ beneath the center key. The chambers were enclosed in sound-attenuating ventilated cases enclosed in a sound-attenuating room. Ventilation fans provided additional masking noise. Each key was backed by an in-line projector. In one box, the projectors were equipped with Kodak Wratten filters and projected red (Wratten No. 92), green (Wratten No. 102), and blue (Wratten No. 75 ) disks with dominant wavelengths at the peak of the pigeon color-naming functions described by Wright and Cumming (1971). In the other box, the projectors were equipped to present three horizontal or three vertical lines, or a white triangle. The centerkey projectors in both boxes were also equipped to display a white disk.

The conditioning chambers were interfaced to a computer located in an adjacent room. Data were recorded on an on-line terminal and on digital cassettes.

Procedure. Two phases of preliminary training were followed by the actual experiment. Phase 1 began with hopper training and subsequent autoshaping. During autoshaping, the response keys were illuminated in the sequence and with the stimuli which would later be the correct pattern for matching-to-sample. (See Roitblat, 1978, for details.)

Each trial in Phase 2 began with the occurrence of an intertrial interval (ITI) during which all stimuli were dark. Following the ITI, the center key was illuminated with a warning signal consisting of a white disk. A single peck to this warning signal caused it to be replaced after $100 \mathrm{msec}$ by the sample stimulus for a fixed 5 -sec duration, during which pecks had no programmed consequences. If the bird failed to peck at the warning signal, it remained illuminated for $5 \mathrm{sec}$, following which the ITI occurred and the trial was re-presented. The sample stimulus was drawn randomly from a pool of three colors-red, green, and blue-or from three pattern stimuli-horizontal or vertical lines, or a triangle.

In order to avoid false responses from perseverative pecks to the warning signal and sample, a minimum of $500 \mathrm{msec}$ was required to elapse following the offset of the sample stimulus without a key depression before the test stimuli were presented.

Following the delay, each of the keys was illuminated with one of the three possible colors or forms. Each stimulus occurred equally often on each key. A single peck to the test stimulus that matched the sample stimulus produced $2.5 \mathrm{sec}$ access to mixed grain. An incorrect choice caused that stimulus to be immediately darkened. The bird was then allowed to choose between the remaining two stimuli. Correct second choices also produced $2.5 \mathrm{sec}$ access to grain, whereas incorrect second choices caused the ITI to be incremented by $30 \mathrm{sec}$. No further correction procedure was used.

Test phase. The procedure used during the final test phase was identical to that used during the second phase of training, with a few exceptions. Each session consisted of 180 trials. Correct choices were reinforced on a random $50 \%$ schedule. Following an incorrect second choice, the correct test stimulus remained illuminated for $2 \mathrm{sec}$. Five sample durations $(100,275,740,2,010$, and $5,460 \mathrm{msec}$ ), chosen to be $1 \mathrm{log}$ unit apart, and two postsample delays ( 500 and $1,000 \mathrm{msec}$ ) were employed in each session. Each trial began with a white warning signal presented on the center key. The warning signal terminated with a single peck and was followed by a sample stimulus presented for a fixed duration. Pecks to the sample stimulus had no programmed consequence. A dark delay interval was presented following sample termination and was subsequently followed by the presentation of three choice stimuli. A failure to choose the correct choice stimulus caused the chosen key to be darkened. A choice was then allowed between the two remaining stimuli. Correct choices were followed by $2.5 \mathrm{sec}$ access to mixed grain in a lighted hopper. Incorrect choices caused the intertrial interval to be increased from its usual 5 to $35 \mathrm{sec}$. Sample duration, delay duration, and location of correct choice key were presented in a random order within each session. In addition, all possible permutations of the three choice stimuli were also presented in random order each day. During the test phase, each bird was run for a total of 20 daily sessions.

\section{Results}

For the purpose of analysis, the data for each bird were averaged over blocks of five sessions. Each of 10 pairs of sample duration and delay duration occurred 90 times within each block of 900 trials. Unless otherwise noted below, each bird's performance was analyzed separately. Figure 1 displays the performance of the five birds as a function of sample duration and delay.

Performance on first choices was analyzed separately from performance on second choices for two reasons: The two models being considered here predict equivalent first-choice performance, but differ in their predictions of performance on second choices, following first-choice errors. Also, chance performance on first choices is .33, while chance accuracy on second choices is .50 , making it difficult to evaluate obtained differences between first-choice and second-choice accuracy.

First-choice ANOVA. The accuracy of first-choice performance was analyzed separately for each bird, with sample duration and delay as factors. For all birds, performance on first choices improved with increases in sample duration [Bird 17: $F(4,30)=$ 33.649, $\mathrm{p}<.001$; Bird 49: $\mathrm{F}(4,30)=34.967, \mathrm{p}<.001$; Bird 71: $F(4,30)=31.777, p<.001 ;$ Bird 60: $F(4,30)$ $=32.276, \mathrm{p}<.001 ;$ Bird $61: \mathrm{F}(4,30)=45.887$, $\mathrm{p}<.001]$. Only Bird 71 showed an effect of delay $[F(1,30)=5.039, p<.032]$. No significant interactions were obtained.

Second-choice ANOVA. To ascertain a general pattern, performance on second choices following first-choice errors was subjected to an analysis of variance with (a) bird, (b) sample duration, and (c) delay as factors. Consistent with the gradual memory model but not with the all-or-none model, combined second-choice performance improved with increases in sample duration $[F(4,150)=35.100$, $\mathrm{p}<.001]$. Delay failed to affect second-choice performance $[F(1,150)=.013]$ and did not interact with any of the other factors. Because the birds differed in their absolute levels of second-choice performance $[F(4,150)=14.226, p<.001]$, however, and because the birds differed in their susceptibility to changes in sample duration $[F(16,150)=3.904, p<.001]$, the second-choice performance for each bird was subjected to a further analysis of variance, with delay and sample duration as factors.

None of the birds were affected by increasing the delay from 500 to $1,000 \mathrm{msec}$, nor were any interactions obtained between delay and sample duration. This failure to find any effect of the brief delays interposed between sample and test stimulus is consistent with the assumption of no forgetting prior to testing memory. 


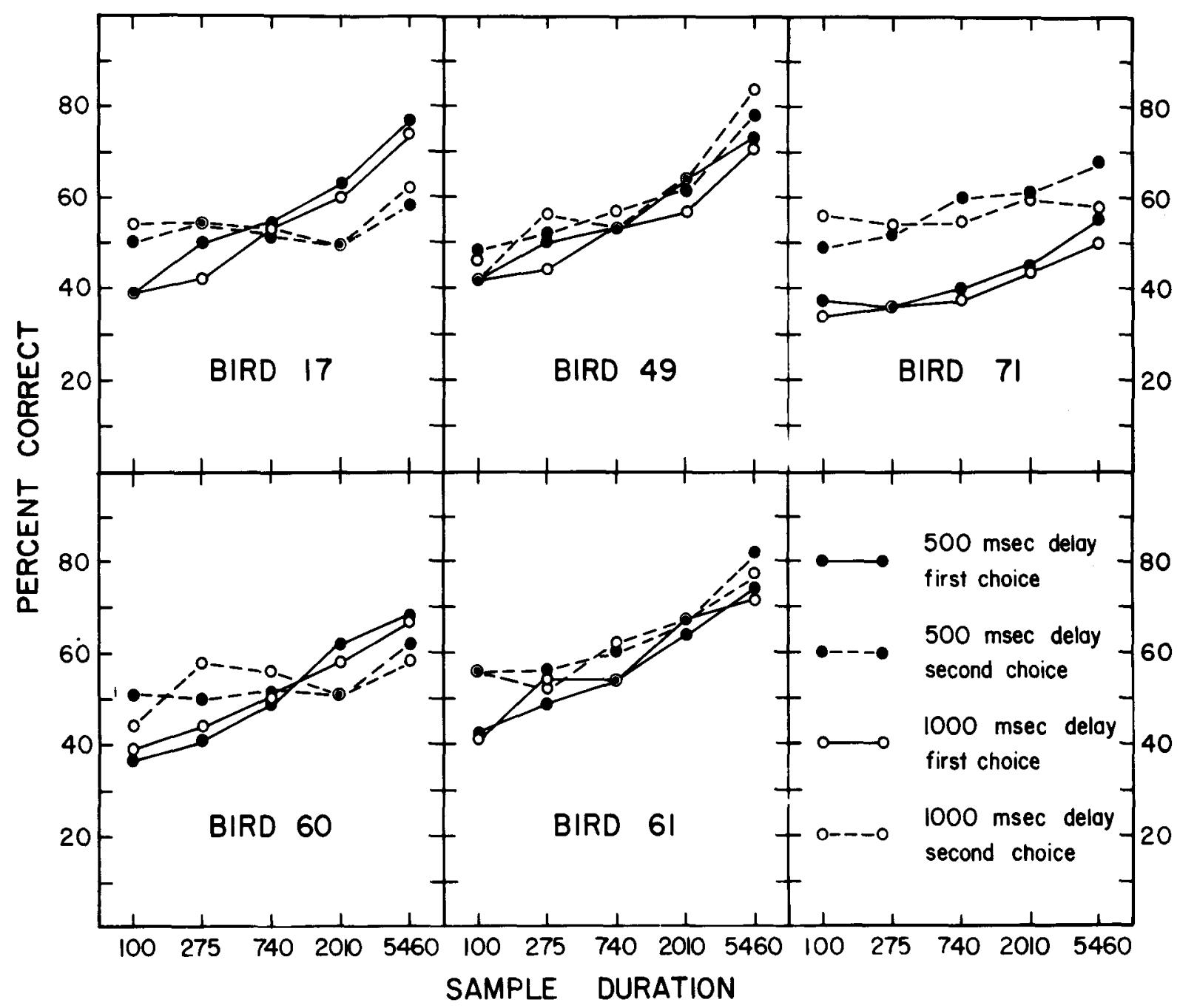

Figure 1. Average levels of performance for each of the five birds in Experiment 1 as a function of sample duration. Note that the abscissa is a log scale.

Again, consistent with the gradual model but not with the all-or-none model, performance on second choices following first-choice errors improved with increases in sample duration for all birds but Bird 17 [Bird 17: $F(4,30)=2.258, p<.086$; Bird 49: $F(4,30)=$ 15.703, $\mathrm{p}<.001 ;$ Bird 71: $\mathrm{F}(4,30)=5.350, \mathrm{p}<.002$ Bird 60: $F(4,30)=5.249, p<.003 ;$ Bird 61: $F(4,30)=$ 16.297, $\mathrm{p}<.001]$.

\section{Discussion}

The major finding of this experiment, as in a number of others (e.g., Maki \& Leith, 1973; Roberts, 1972; Roberts \& Grant, 1974), is an improvement in performance with increases in sample duration. Apparently, during sample presentation, the bird accumulates information about the sample that permits increasingly accurate choices. The change from inadequate to adequate levels of information could occur as a result of a sudden shift from having no information to having information, but the improvement in secondchoice performance obtained in this experiment is consistent with the hypothesis that the change is more gradual in nature, or at least that it depends on more factors than a simple all-or-none encoding process.

The results obtained in the present experiment with pigeons may not be general to other species. For example, D'Amato and Worsham (1972) trained capuchin monkeys on a DMTS task and failed to find any effect of sample duration. More recently, however, Devine, Jones, Neville, and Sakai (1977) have reported that rhesus monkeys do show an improvement in performance with increasing sample duration (see, also, Herzog, Grant, \& Roberts, 1976). D'Amato (1973) accounts for the data obtained by D'Amato and Worsham (1972) by suggesting that "perhaps because of the considerable experience [the] subject [in that study had] with the sample set, each stimulus was a highly organized configuration which could be 
identified even with partial information, just as a human observer can identify a triangle at exposure times too brief for him to perceive the entire figure" (D'Amato, 1973, p. 246). D'Amato's animals were more highly trained with the sample and test stimuli than the animals in the study by Devine et al. (1977). Though the experiment has not apparently been reported yet, level of training seems to be the critical variable involved in accounting for the differences in findings with monkeys.

Pigeons, on the other hand, despite experience derived from hundreds of thousands of trials (Maki \& Leith, 1973), do not seem to be able to do as well with short-duration samples as with long. This difference implies that monkeys and pigeons may be very different in the means they use to perform in DMTS tasks. It may be the case that pigeons remember which sample they have just seen by slowly accumulating a representation of the information contained in the sample, while monkeys may remember when each of the various sample stimuli was presented by suddenly setting a time tag. Such a hypothesis is in keeping with intuitive notions regarding the relative cognitive capacities of monkeys vs. pigeons but has yet to be tested. Nevertheless, the data of the first experiment seem to indicate that pigeons form a representation of the information in the sample stimulus in a rather slow and gradual manner.

\section{EXPERIMENT 2}

The all-or-none model failed to predict the data of Experiment 1 because it recognizes only a single error state. While the data from the first experiment allowed us to rule out this model, they did not rule out all discrete state models. For example, an "inattention" model involving two error-producing states, each operating in an all-or-none fashion, produces predictions that are consistent with the results obtained in Experiment 1 (Roitblat, 1978).

The inattention model is based on the assumption that performance on a DMTS trial represents a combination of two processes. On some proportion of the trials, the bird is assumed to accurately encode the information in the sample stimulus in an all-or-none fashion. On some independent proportion of the trials, however, some nonmemorial process controls responding. That is, on some trials the subject chooses among the comparison stimuli without regard to the sample stimulus, either because it fails to attend to the comparison stimuli or because they elicit, by virtue of their close association with reinforcement, autoshaped or classically conditioned pecks (Roberts \& Grant, 1978). It is also assumed that attention to the test stimuli for the first choice is independent of attention to the test stimuli for the second choice. Non- independence would add a free parameter and lower the predicted second-choice accuracy.

In many ways the inattention model is fairer as an alternative to the gradual model in that both, for example, recognize that there may be two processes controlling DMTS performance. For the gradual model, each element in memory is encoded and retrieved incrementally (i.e., not all-or-none). For the inattention model, it is assumed that memory consists of only a single memory element which is encoded and retrieved as a whole, both in an all-or-none fashion. Both models predict above chance secondchoice responding when the opportunity to make a second choice is contingent on the occurrence of a first-choice error. The two models differ, however, in their predicted distributions of first-choice errors. For the inattention model, it is assumed that errors result either from a failure to encode the sample stimulus or from a failure to attend to the comparison stimuli when making a choice among them. In either case, error responses should be independent of the sample. For the gradual model, on the other hand, errors can result not only from a complete lack of information, but also from partial information. Therefore, the gradual model is consistent with distributions of error responses that contain some information about the sample. Experiment 2 was conducted in order to determine whether or not first-choice errors were distributed randomly with respect to the sample stimulus.

\section{Method}

The subjects, apparatus, and stimuli were identical to those used in Experiment 1. Each bird was exposed to five sessions (180 trials each). A single postsample delay duration $(500 \mathrm{msec})$ was used on all trials for all birds. Sample duration was held constant for all trials at a value determined in Experiment 1 to produce approximately $70 \%$ correct first-choice performance. Sample durations were: 1,500 msec for Birds 17 and 49, 5,000 msec for Bird 71, and 2,000 msec for Birds 60 and 61 .

\section{Results and Discussion}

Table 1 presents the observed and expected frequency distributions for first-choice errors. Each row in the table corresponds to the sample presented on a trial; each column corresponds to the response that occurred. Each sample was presented 300 times to each bird.

The expected distributions were derived on the assumption that error responses were distributed independently of the sample stimulus, depending only on the average distribution of responses to the three available stimuli. Expected cell frequencies were computed by calculating the proportion of responses that were emitted to the relevant comparison stimulus (its column total including correct responses) divided by the total number of responses to the two stimuli, which, for that sample, would have been errors. This proportion was rounded to two decimal places and 
Table 1

Frequency Distribution of First-Choice Errors

\begin{tabular}{|c|c|c|c|c|c|c|}
\hline & \multicolumn{3}{|c|}{ Observed } & \multicolumn{3}{|c|}{ Expected } \\
\hline & A & B & $\mathrm{C}$ & A & B & $\mathrm{C}$ \\
\hline & \multicolumn{6}{|c|}{ Bird $17\left[\chi^{2}(1)=1.72\right]$} \\
\hline A & 0 & 30 & 24 & .0 & 29.2 & 24.8 \\
\hline B & 37 & 0 & 51 & 50.2 & .0 & 37.8 \\
\hline \multirow[t]{2}{*}{$\mathrm{C}$} & 56 & 63 & 0 & 63.1 & 55.9 & .0 \\
\hline & \multicolumn{6}{|c|}{ Bird $49\left[x^{2}(1)=22.46^{*}\right]$} \\
\hline A & 0 & 127 & 14 & .0 & 76.1 & 4.9 \\
\hline B & 13 & 0 & 59 & 24.5 & .0 & 47.5 \\
\hline \multirow[t]{2}{*}{ C } & 2 & 36 & 0 & 11.8 & 26.2 & .0 \\
\hline & \multicolumn{6}{|c|}{ Bird $71\left[\chi^{2}(1)=1.22\right]$} \\
\hline A & 0 & 64 & 60 & .0 & 54.6 & 69.4 \\
\hline B & 49 & 0 & 79 & 52.5 & .0 & 75.5 \\
\hline \multirow[t]{2}{*}{$\mathrm{C}$} & 27 & 52 & 0 & 37.1 & 41.9 & .0 \\
\hline & \multicolumn{6}{|c|}{ Bird $60\left[x^{2}(1)=10.47^{*}\right]$} \\
\hline A & 0 & 64 & 60 & .0 & 54.6 & 69.4 \\
\hline B & 27 & 0 & 91 & 54.3 & .0 & 63.7 \\
\hline \multirow[t]{2}{*}{ C } & 14 & 52 & 0 & 35.0 & 31.0 & .0 \\
\hline & \multicolumn{6}{|c|}{ Bird $61\left[\chi^{2}(1)=23.58^{*}\right]$} \\
\hline A & 0 & 17 & 14 & .0 & 14.0 & 17.0 \\
\hline B & 14 & 0 & 106 & 56.4 & .0 & 63.6 \\
\hline $\mathrm{C}$ & 14 & 74 & 0 & 45.8 & 42.2 & .0 \\
\hline
\end{tabular}

Note-Each row corresponds to the sample presented on a trial, and each column corresponds to the response that occurred. The major diagonal is always zero because these stimulus-response pairs were not errors. Each sample occurred 300 times for each bird. $\quad * \quad p .01$.

multiplied by the total number of errors following the relevant sample (the row totals excluding correct responses). For example, the expected number of "B" errors following sample "A" for Bird 17 was computed as follows: Including the correct $B$ responses (i.e., those $B$ responses made following sample B), Bird 17 made a total of 305 B responses. Analogously, 256 " C " responses were also produced. $B$ responses therefore corresponded to $54 \%$ of the $B$ and $C$ responses made $[305 /(305+256)]$ and so could therefore be expected to comprise $54 \%$ of the errors following sample $A$, or $54 \%$ of the 54 errors. The expected cell frequency, then, represents the number of responses to be expected if errors are distributed independently of the sample stimulus, depending only on overall bias to respond to one or another of the three comparison stimuli.

For three of the five birds serving in Experiment 2, the distribution of first-choice errors was significantly different from that expected on the basis of the inattention model. In addition, all birds but Bird 17 reached second-choice levels of accuracy significantly above chance. These results provide further support for the gradual model in preference to either of the two discrete-state models considered (but not necessarily all discrete-state models).

Systematic error distributions imply that the bird had some information about the sample even when making error choices. The converse, however, is not necessarily true. Nonsystematic error distributions do not necessarily imply a complete lack of information because there was nothing explicitly forcing one type of error to be more likely than another except on the basis of overall response biases (column totals). For example, since the color stimuli used in this experiment were chosen to be in the middle of one of the pigeon's color names (Wright \& Cumming, 1971), it might be expected that one day the pigeon might be biased to choose "red" when making an error response based on partial information about a green sample and on another day choose "blue" based on the same information. The result, when averaged across days, would be a random distribution of first-choice errors.

When the results of Experiments 1 and 2 are considered together, it seems most reasonable to view the pigeon's matching-to-sample performance as a result of gradually building up a coded representation of the sample stimulus for use in choosing the correct match. Experiment 3 was intended to provide some insight into the particular coding process that is being used.

\section{EXPERIMENT 3}

Another aspect of Roberts' (1972; Roberts \& Grant, 1976) model is the assumption that the pigeon's representation of the information contained in the sample is in the form of a copy of the sample. While this is the most obvious and straightforward representation, there are several lines of evidence indicating that this hypothesis is too simple to adequately account for pigeon DMTS performance (Roitblat, 1978). The most important difficulty arises from the observation that similarity between the sample and test stimuli is neither sufficient nor necessary to produce matching. For example, following initial training with one set of three sample and test stimuli, Cumming, Berryman, and Cohen (1965) failed to find transfer to a novel pair of sample and test stimuli even though the novel sample and test stimuli were identical to each other. Second, pigeons are able to perform symbolic matching-to-sample in which the relationship between the comparison and sample stimuli is arbitrary and not dependent on physical similarity (Carter \& Eckerman, 1975; Cohen, Looney, Brady, \& Acuella, 1976). Thus, pigeon matching performance seems not to be based on a single rule of the form "pick the test item most similar to the sample stimulus whose copy appears in memory," but is more likely based on some sort of multiple rule model of the form "If sample A occurs, peck test item X" (Carter, 1971; Carter \& Eckerman, 1975; Carter \& Werner, 1978; Cumming \& Berryman, 1965). 
According to this latter view, the subject maintains one rule in memory for each sample or each sample-test pair. On every trial, the bird must remember which sample stimulus occurred on that trial. By applying the rule to the most recent sample stimulus, the correct choice can be determined. This translation process, that is, the application of the rule transforming the sample to the correct test stimulus, must occur sometime between the onset of the sample stimulus and the time a choice is made.

The information could be maintained in any of at least three forms. One possibility is that the bird could remember a more or less direct copy of the sample stimulus during the delay and apply the rule to the representation only when the choice stimuli are presented. This alternative is a restatement of the simple "copy" hypothesis proposed by Roberts (1972), with the addition of the assumption that choices are made on the basis of a mapping rule rather than on the basis of a more direct comparison of the sample and test stimuli. According to this alternative, the mapping rule is applied late in the interval between sample and test, just prior to the occurrence of a choice. For most of the delay, then, the memory is coded as a "copy" of the sample stimulus.

As a second alternative, the bird could immediately translate the sample stimulus into a form isomorphic with the correct test stimulus and maintain that representation in memory during the delay. Therefore, the information present in the sample is maintained for most of the delay interval in a form similar to the upcoming correct comparison stimulus. A suggestion to that effect has been offered by Cumming and Berryman (1965).

A third possibility is that the information contained in the sample is quickly translated according to some mapping rule into some other intermediate form (e.g., a time tag), maintained in that form for most of the delay, and then finally used as the input to a second mapping rule which produces the choice response. In humans, such an intervening form would likely be a verbal label. In animals, this intermediate form might be some kind of "time tag" (D'Amato, 1973; Mason \& Wilson, 1974; Mishkin \& Delacour, 1975; Winograd, 1971; Worsham, 1975).

The methodology used to discriminate among these three alternative memory codes is based on the analysis of confusion errors. As more confusions occur (e.g., with increasing intervals between sample and comparison stimulus presentations), they should be more likely between similar than between dissimilar items (Beals, Krantz, \& Tversky, 1968).

The particular technique employed in Experiment 3 is based on an experiment by Conrad (1964) in which human subjects were asked to remember visually presented consonants. When errors were produced in this experiment, they tended to be acoustically similar to the correct item and not visually similar. This suggested to Conrad that the subjects stored the representations of the visually presented items in an acoustic code.

A situation analogous to Conrad's was set up for Experiment 3 through the use of symbolic DMTS. The test stimuli in symbolic DMTS vary along a dimension different from the nominal dimension along which the sample stimuli vary. The mapping of sample stimuli onto comparison stimuli is therefore arbitrary. For pigeons, there appears to be little difference between symbolic DMTS and identity DMTS in terms of speed of acquisition or asymptotic level of performance (Carter \& Eckerman, 1975; Cohen, Looney, Brady, \& Acuella, 1976).

In Experiment 3, color sample stimuli were followed by line-orientation test stimuli, and line-orientation sample stimuli (for a different bird) were followed by color test stimuli. As in the first two experiments, three samples and test stimuli were used, one sample and three comparison stimuli appearing on each trial. In this experiment, however, the sample and comparison stimuli were chosen so that similar comparison stimuli corresponded to dissimilar sample stimuli and dissimilar comparison stimuli corresponded to similar sample stimuli. With this particular configuration of stimuli, it was hoped to discriminate among memory codes used by the birds.

\section{Method}

Subjects. Three experimentally naive Silver King pigeons served as subjects. As in the first two experiments, they were maintained at between $75 \%$ and $80 \%$ of their free-feeding weights. Birds 51 and 52 were trained with color samples and line orientation tests; Bird 62 was trained with line orientation samples and color tests.

Apparatus. Experimental sessions were conducted in a standard three-key operant chamber similar to those used in Experiment 1. Each key was backed by an in-line projector equipped to present three color stimuli-blue (Wratten filter No. 75), orange (Wratten filter No. 22), and red (Wratten filter No. 25)-and three lineorientation stimuli-vertical $(0 \mathrm{deg})$, slant $(12.5 \mathrm{deg})$, and horizontal ( $90 \mathrm{deg}$ )-consisting of two dark lines, $3 \mathrm{~mm}$ wide, centered about a radius of the key and presented against a white surround. Note that two of the sample stimuli are very similar to one another (red and orange) and that these correspond to two test stimuli that are rather dissimilar (12.5 and $90 \mathrm{deg})$. At the same time, two of the adjacent sample stimuli are dissimilar (blue and orange) and correspond to two test stimuli that are similar ( 0 and $12.5 \mathrm{deg}$ ). The center key was also equipped to display a white disk.

Procedure. Preliminary training was identical to that described in the first experiment. During experimental sessions, each trial began with the illumination of the center key with a white disk (until a peck occurred or until 5 sec had elapsed). If a peck occurred during the tenure of this warning signal, it was replaced. $100 \mathrm{msec}$ later, by the sample stimuli. For two of the birds, the sample stimuli were drawn from a pool of three color stimuli. For the third bird, the samples were drawn from a pool of three line-orientation stimuli.

The experiment was conducted in two phases (15 sessions each). The phases differed in the sample durations used. During Phase 1, 10- and 1-sec samples and 250-, 500-, 1,000-, 2,000-, and 4,000-msec delays were used. During Phase 2, 10- and 5-sec sample durations and 350-, 700-, 1,400-, 2,800-, and 5,600-msec delay durations were used. Color samples were followed by line orientation tests, 
and line orientation samples were followed by color test comparison stimuli. Each bird, however, received only one kind of samplecolors for Birds 51 and 52 and lines for Bird 62. All combinations of sample duration and delay occurred equally often in random order during each 180-trial session. Correct choices were reinforced with $2.5 \mathrm{sec}$ access to mixed grain. Following incorrect first choices, however, the chosen stimulus was darkened and a second choice was allowed between the remaining two test stimuli. Correct second choices were also reinforced. Incorrect second choices resulted in an increment of the ITI from its standard $5 \mathrm{sec}$ to $35 \mathrm{sec}$.

\section{Results and Discussion}

Before discussing the data relevant to the nature of the memory code, some comparisons between the data obtained in this experiment and the data obtained from the first experiment are in order. The all-or-none model predicts that second-choice accuracy should be independent of first-choice accuracy. The gradual model, on the other hand, predicts that second-choice accuracy should improve with increasing first-choice accuracy. To the extent that forgetting occurs, it should cause the results to more closely resemble those predicted by the all-or-none model.

Consistent with the gradual model, positive correlations were obtained between performance on first choices and performance on second choices following first-choice errors (Bird 51: $\mathrm{r}=.5167, \mathrm{p}<.001$; Bird 52: $\mathrm{r}=.6230, \mathrm{p}<.001$; Bird 62: $\mathrm{r}=.3624$, $p<.005$ ). The results from this experiment are thus seen to replicate those obtained in the first experiment.

Analysis of variance. First-choice performance levels for the three subjects in Experiment 2 are presented in Figure 2. Each bird's first-choice performance was subjected to an analysis of variance preliminary to an examination of the memory code. Data from Phase 1 and Phase 2 were combined.

First-choice performance improved for all three birds with increases in sample duration [Bird 51: $F(2,40)=70.605, p<.001 ;$ Bird 52: $F(2,40)=72.395$, $\mathrm{p}<.001$; Bird 62: $\mathrm{F}(2,40)=48.790, \mathrm{p}<.001]$. Performance was also significantly affected by the length of the delay interval following sample termination [Bird 51: $F(9,40)=5.286, p<.001 ;$ Bird 52: $F(9,40)$ $=22.697, \mathrm{p}<.001$; Bird 62: $F(9,40)=4.645$, $\mathrm{p}<.001$ ]. All three birds performed best with postsample delay durations of $700 \mathrm{msec}$, a finding similar to that obtained by Roitblat, Greene, and Riley (Note 1; Riley \& Roitblat, 1978).

Overall, there was surprisingly little effect of delay. The typical finding is that performance declines as a negatively accelerating function of delay (e.g., Grant, 1975; Roberts, 1972). With sample durations of the magnitude used in this experiment, Roberts and Grant (1974) found a significant decline in performance with a $1-\mathrm{sec}$ delay. The relatively small effect of delay obtained in this experiment may be due to the training procedure used. Typically, training with DMTS starts with either simultaneous presentation of the sample and test stimuli or with a 0 -sec delay. Training in this experiment began with a .5 -sec delay

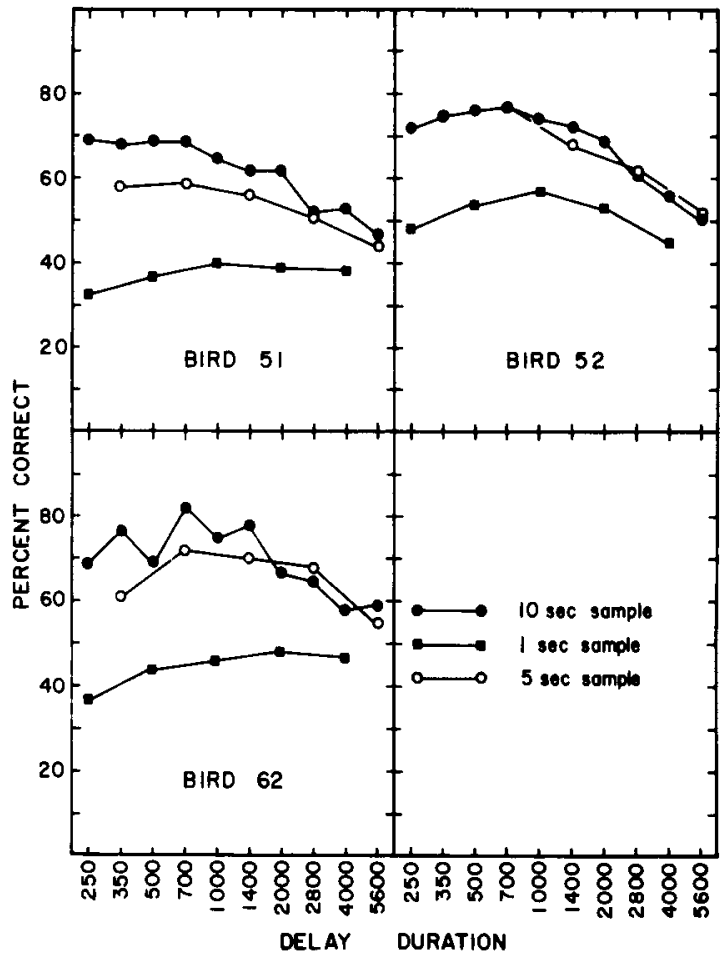

Figure 2. Average levels of performance for the five birds in Experiment 2 as a function of delay duration. Note that the abscissa is a log scale.

between sample of fset and test onset. Perhaps during this early training the bird developed rehearsal techniques which controlled its later performance.

Memory code. If the birds are remembering an elaborated code of the sample stimulus, then more similar samples will be more likely confused with increasing delays than will more dissimilar samples. Similarly, if the bird is remembering the test stimuli, then the rate of confusions between similar test stimuli should increase more rapidly with increasing delay than should the rate of confusions between dissimilar test stimuli. For Birds 51 and 52, sample A (blue) and sample B (orange) were dissimilar to one another, while sample B and sample $C$ (red) were similar to one another. At the same time, test items A ( $0 \mathrm{deg})$ and B (12.5 deg) were similar to one another relative to test stimuli $B$ and $C(90 \mathrm{deg})$. Therefore, as delay increases, the birds should be increasingly inclined to confuse $B$ and $C$ if they are remembering the sample stimuli and to confuse $A$ and $B$ if they are remembering the test stimuli. Mainly for convenience, discriminal distances, rather than confusion indices, were computed. The two are exactly equivalent; one represents closeness and the other represents distance. For Birds 51 and 52, the ratio of the discriminal distance between the A and B stimuli, on the one hand, and the $B$ and $C$ stimuli, on the other, should increase with increasing delay if the birds are remembering the 
Table 2

The Form of the Sample-Response Matrix Used to Compute Discriminal Distances

\begin{tabular}{lccc}
\hline & \multicolumn{3}{c}{ Response } \\
\cline { 2 - 4 } Sample & 0 & 12.5 & 90 \\
\hline BLUE & $\mathrm{R}$ & $\mathrm{S}$ & $\mathrm{T}$ \\
ORANGE & $\mathrm{U}$ & $\mathrm{V}$ & $\mathrm{W}$ \\
RED & $\mathrm{X}$ & $\mathrm{Y}$ & $\mathrm{Z}$ \\
\hline
\end{tabular}

Note-The values $0,12.5$, and 90 refer to the line orientations present on the comparison stimuli. In actually computing discriminal distances, the frequency with which each response was made would be substituted for the letter in the present table (see text)

sample stimuli and decrease if they are remembering the test stimuli. On the other hand, the sample and test stimuli were exchanged for Bird 62. Samples A $(0 \mathrm{deg})$ and $\mathrm{B}(12.5 \mathrm{deg})$ were similar to one another relative to samples $B$ and $C(90 \mathrm{deg})$. Test stimuli $A$ and $B$ were dissimilar relative to test stimuli B and $C$. This bird's discrimination ratio should increase therefore if it is remembering the comparison stimuli and decrease if its memory code is similar to the sample stimuli. To test these hypotheses, the discriminal distances were computed and subjected to a regression analysis.

The discriminal distances were computed as follows. Each block of five sessions produced a choice-bysample matrix, such as appears in Table 2, for each combination of sample duration and delay. Thus, each block of sessions produced 10 matrices from which distance indices were derived. Three discriminal distances were computed: distance $\mathrm{AB}=1-(\mathrm{S}+\mathrm{U}) / 60$; distance $\mathrm{BC}=1-(\mathrm{W}+\mathrm{Y}) / 60$; distance $\mathrm{AC}=$ $1-(\mathrm{T}+\mathrm{X}) / 60$.

These distance indices are presented in Table 3. For Birds 51 and 52, the line relating the $\mathrm{AB} / \mathrm{BC}$ ratio to delay duration had a negative slope, indicating a decrease in the ratio with increasing delay duration [Bird 51: slope $=-.048, \mathrm{~F}(1,58)=20.945, \mathrm{p}<.001$; Bird 52: slope $=-.047, \mathrm{~F}(1,58)=12.561, \mathrm{p}<.001]$. In other words, for these two birds, confusions between similar test items increased faster than did confusions between similar samples, suggesting that these birds were remembering the test items during the delay. Bird 62, for which the sample and test stimuli were reversed, presented an upward trend, also consistent with a memory for the test stimuli, but this trend was not significant [slope $=.0153, F(1,58)=1.452$, $\mathrm{p}<.233$ ]. The most straightforward conclusion to be drawn from these results is that the birds remembered the test stimulus which was eventually to be the correct choice. Apparently, during the sample presentation they encoded the information in the sample stimulus by forming a representation similar to the test stimulus which was to be reinforced.

\section{GENERAL DISCUSSION}

The results of these experiments suggest that, in the performance of DMTS, pigeons gradually build up memory representations which have properties isomorphic with the correct test stimulus. Apparently, this is a somewhat complex process. The bird must identify the sample stimulus being presented, query long-term memory to retrieve the appropriate mapping rule, and then generate the representation of the correct test stimulus. Perhaps the gradual nature of this process is involved in generating multiple copies of the test representation, each encoded in an all-ornone manner. For example, the bird's memory system might consist of a large number of memory elements which are sampled at some rate during a sample presentation. As a memory element is sampled, it is labeled with a representation of the test stimulus. If one assumes that there will be some encoding variability, that is, that the stored representation will vary somewhat around the intended representation, and assumes that only a portion of the elements are sampled during the test, then one has a theory that accounts fairly well for the obtained data. Memory increases gradually (or at least in very small steps) in strength and produces confusions that reflect the similarities among the test stimuli (see Neimark \& Estes, 1967).

The evidence reviewed and reported here does not support the kind of memory model proposed by Roberts (1972; Roberts \& Grant, 1976), which assumes that subjects base their DMTS performance on the comparison of the available test stimuli with a mnemonic copy of the sample. It appears, instead, that pigeons utilize a more elaborate and complex means to represent the information presented in the sample. The bird's inability to perform the DMTS task with distinctively novel sample and test stimuli indicates that the increase in trace strength is not due simply to an increase in clarity of the image of the sample. The pigeon appears to engage in an active coding process during sample presentation in which the features of the sample stimulus are replaced with the features of the correct test stimulus. This representation appears to vary along dimensions that are isomorphic with the dimensions along which the test stimuli vary. In other words, pigeons appear to store the information about the sample, not in terms of an image of the presented sample, but more similarly to an image of the correct choice stimulus.

The results of these experiments also suggest that pigeons perform DMTS on the basis of a multiple rule model. This conclusion is contrary to that reached by Maki, Riley, and Leith (1976) from their study of DMTS involving unidimensional and bidimensional sample and test stimuli. They reported that performance with unidimensional (element) sample stimuli was always superior to performance with bidimen- 
Table 3

Discriminal Distance

\begin{tabular}{|c|c|c|c|c|c|c|c|c|c|c|}
\hline \multirow[b]{2}{*}{ Distance } & \multicolumn{5}{|c|}{ Phase 1 Delay (in Milliseconds) } & \multicolumn{5}{|c|}{ Phase 2 Delay (in Milliseconds) } \\
\hline & 250 & 500 & 1000 & 2000 & 4000 & 350 & 700 & 1400 & 2800 & 5600 \\
\hline & \multicolumn{10}{|c|}{ Bird 51} \\
\hline & \multicolumn{5}{|c|}{ 10-Sec Sample } & \multicolumn{5}{|c|}{ 10-Sec Sample } \\
\hline $\mathrm{AB}$ & .79 & .78 & .75 & .72 & .63 & .78 & .77 & .75 & .70 & .630 \\
\hline BC & .82 & .83 & .79 & .79 & .78 & .79 & .82 & .78 & .79 & .770 \\
\hline $\mathrm{AC}$ & .91 & .96 & .93 & .91 & .88 & .95 & .94 & .84 & .86 & .800 \\
\hline \multirow[t]{2}{*}{$\mathrm{AB} / \mathrm{BC}$} & .97 & .94 & .95 & .92 & .82 & .99 & .95 & .96 & .88 & .820 \\
\hline & \multicolumn{5}{|c|}{ 1-Sec Sample } & \multicolumn{5}{|c|}{ 5-Sec Sample } \\
\hline$A B$ & .62 & .65 & .67 & .61 & .58 & .72 & .74 & .71 & .66 & .610 \\
\hline $\mathrm{BC}$ & .68 & .67 & .68 & .75 & .76 & .77 & .76 & .77 & .73 & .740 \\
\hline & .69 & .74 & .76 & .67 & .72 & .82 & .88 & .85 & .85 & .800 \\
\hline \multirow[t]{3}{*}{$\mathrm{AB} / \mathrm{BC}$} & .92 & .97 & .98 & .82 & .78 & .94 & .98 & .92 & .90 & .830 \\
\hline & \multicolumn{10}{|c|}{ Bird 52} \\
\hline & \multicolumn{5}{|c|}{ 10-Sec Sample } & \multicolumn{5}{|c|}{ 10-Sec Sample } \\
\hline$A B$ & .86 & .86 & .90 & .85 & .72 & .83 & .84 & .85 & .79 & .720 \\
\hline $\mathrm{BC}$ & .76 & .83 & .77 & .81 & .86 & .87 & .83 & .80 & .78 & .780 \\
\hline & .94 & .95 & .95 & .89 & .80 & .95 & .98 & .94 & .87 & .800 \\
\hline \multirow[t]{2}{*}{$\mathrm{AB} / \mathrm{BC}$} & 1.14 & 1.04 & 1.11 & 1.05 & .84 & .96 & 1.02 & 1.07 & 1.02 & .920 \\
\hline & \multicolumn{5}{|c|}{ 1-Sec Sample } & \multicolumn{5}{|c|}{ 5-Sec Sample } \\
\hline$A B$ & .71 & .76 & .78 & .77 & .66 & .85 & .84 & .80 & .81 & .690 \\
\hline $\mathrm{BC}$ & .68 & .76 & .73 & .81 & .76 & .83 & .86 & .81 & .77 & .810 \\
\hline & .83 & .79 & .85 & .72 & .77 & .94 & .96 & .91 & .85 & .790 \\
\hline \multirow[t]{3}{*}{$\mathrm{AB} / \mathrm{BC}$} & 1.05 & .99 & 1.08 & .96 & .87 & 1.02 & .98 & .98 & 1.06 & .840 \\
\hline & \multicolumn{10}{|c|}{ Bird 62} \\
\hline & \multicolumn{5}{|c|}{ 10-Sec Sample } & \multicolumn{5}{|c|}{ 10-Sec Sample } \\
\hline $\mathrm{AB}$ & .77 & .81 & .78 & .78 & .74 & .88 & .86 & .82 & .80 & .780 \\
\hline BC & .87 & .87 & .91 & .84 & .76 & .84 & .91 & .92 & .80 & .750 \\
\hline $\mathrm{AC}$ & .91 & .89 & .93 & .88 & .87 & .93 & .96 & .92 & .88 & .850 \\
\hline \multirow[t]{2}{*}{$\mathrm{AB} / \mathrm{BC}$} & .89 & .94 & .87 & .93 & 1.00 & 1.05 & .95 & .89 & 1.00 & 1.032 \\
\hline & \multicolumn{5}{|c|}{ 1-Sec Sample } & \multicolumn{5}{|c|}{ 5-Sec Sample } \\
\hline $\mathrm{AB}$ & .66 & .67 & .68 & .70 & .71 & .76 & .79 & .75 & .80 & .720 \\
\hline & .69 & .71 & .74 & .72 & .72 & .81 & .88 & .87 & .83 & .770 \\
\hline AC & .72 & .78 & .76 & .79 & .78 & .86 & .91 & .93 & .88 & .820 \\
\hline $\mathrm{AB} / \mathrm{BC}$ & .96 & .96 & .93 & .98 & .99 & .93 & .89 & .86 & .97 & .950 \\
\hline
\end{tabular}

Note-Table entries are averages of obtained measures for each of three blocks (five sessions per block). Thus, the ratio entries may not correspond to the ratio of the distance entries. Samples and tests are reversed for Bird 62 relative to Birds 51 and 52 , so an equivalent change from a theoretical point of view is in the opposite direction for this bird.

sional (compound) stimuli, even when the test stimuli were bidimensional, redundant, and relevant. With redundant relevant test stimuli, the multiple rule model, they suggested, should produce the same level of performance following unidimensional samples as following bidimensional samples because, in both cases, only a single rule is necessary to specify the correct response. As the data did not conform to this prediction, they concluded that pigeons do not maintain the information from the sample in terms of remembering each of the applicable rules for the stimuli occurring in that sample.

Their experiment may not have been an appropriate test of the multiple rule model, however. First, the version of the multiple rule model presented here suggests that the rules themselves are not maintained in short-term memory (STM) during the actual performance of the DMTS task. Second, each of the experiments reported in that paper involved the same two birds, which had also served in several previous experiments in which unidimensional and bidimensional sample stimuli were always followed by unidimensional tests. If the assumption of the present version of the multiple rule model is correct, that rules are like habits, then it may be difficult to produce a change in the bird's memory of the rules without explicit extinction and retraining. No new rules were necessary in their experiment for the bird to maintain a comparable level of performance. Repeating the experiment with more naive animals would likely result in very different findings and further support a multiple rule model. 


\section{REFERENCE NOTE}

1. Roitblat, H. L., Greene, L. H., \& Riley, D. A. Perception of brief visual stimuli by pigeons. Paper presented at the meeting of the Western Psychological Association, Los Angeles, April 1976.

\section{REFERENCES}

Beals, R., Krantz, D. H., \& Tversky, A. Foundations of multidimensional scaling. Psychological Review, 1968, 75, $127-142$.

Bower, G. H. Application of a model to paired-associate learning. Psychometrika, 1961, 26, 255-280.

Bush, R. R.. \& Mosteller, F. Stochastic models for learning. New York: Wiley, 1955.

Carter, D. E. Acquisition of a conditional discrimination: A comparison of matching-to-sample and symbolic matching. Unpublished doctoral dissertation, Columbia University, 1971.

Carter, D. E., \& Eckerman, D. A. Symbolic matching by pigeons: Rate of learning complex discriminations predicted from simple discriminations. Science, 1975, 187, 662-664.

Carter, D. E., \& Werner, T. J. Complex learning and information processing by pigeons: A critical analysis. Journal of the Experimental Analysis of Behavior, 1978, 29, 565-601.

Cohen, L. R., Looney, T. A., Brady, J. H., \& Acuella, A. F. Differential sample response schedules in the acquisition of conditioned discriminations by pigeons. Journal of the Experimental Analysis of Behavior, 1976, 26, 301-314.

Conrad, R. Acoustic confusions in immediate memory. British Journal of Psychology, 1964, 55, 75-83.

Cumming, W. W., \& Berryman, R. The complex discriminated operant: Studies of matching-to-sample and related problems. In D. I. Mostofsky (Ed.), Stimulus generalization. Stanford, Calif: Stanford University Press, 1965

Cumming, W. W.. Berryman, R., \& Cohen, L. R. Acquisition and transfer of zero-delay matching. Psychological Reports, $1965,17,435-445$

D'Amato, M. R. Delayed matching and short-term memory in monkeys. In G. H. Bower (Ed.), The psychology of learning and motivation: Advances in research and theory (Vol. 7). New York: Academic Press, 1973.

D'Amato, M. R., \& Worsham, R. W. Delayed matching in the capuchin monkey with brief sample durations. Learning and Motivation, 1972, 3, 304-312.

Devine, J. V., Jones, L. C., Neville, J. W., \& Sakai, D. J. Sample duration and type of stimuli in delayed matching-tosample in rhesus monkey. Animal Learning \& Behavior, 1977 , $5,57-62$.

Estes, W. K. Statistical theory of spontaneous recovery and regression. Psychological Review, 1955, 62, 145-154.

Fartiling, G. W., Wagner, J. M., Gilmour, S., \& Waxman, H. M. Short term memory and information processing in pigeons. Learning and Motivation, 1977, 8, 520-532.

Grant, D. S. Proactive interference in pigeon short-term memory. Journal of Experimental Psychology: Animal Behavior Processes, 1975, 104, 207-220.

Grant, D. S., \& Roberts, W. A. Sources of retroactive inhibition in pigeon short-term memory. Journal of Experimental Psychology: Animal Behavior Processes, 1976, 2, 1-16.

Herzog, H. L., Grant, D. S., \& Robrints, W. A. Effects of sample duration and spaced repetition upon delayed matchingto-sample in monkeys (Macaca arctoides and Saimiri sciureus). Animal Learning \& Behavior, 1977, 5, 347-354.

Mackintosh, N. J. A theory of attention: Variations in the associability of stimuli with reinforcement. Psvchological Review, 1975, 82, 276-298.

Mакі, W. S., JR., \& Ltitr, C. R. Shared attention in pigcons. Journal of the Experimental Analvsis of Behavior, 1973, 19. 345-349.
Maki, W. S., JR., Riley, D. A., \& Leiti, C. R. The role of test stimuli in matching to compound sample in pigeons. Animal Learning \& Behavior, 1976, 4, 13-21.

Mason, M., \& Wilson, M. Temporal differentiation and recognition memory for visual stimuli in rhesus monkeys. Journal of Experimental Psychology, 1974, 103, 383-390.

Mishkin, M., \& Delacour, J. An analysis of short-term visual memory in the monkey. Journal of Experimental Psychology: Animal Behavior Processes, 1975, 1, 326-334.

Neimark, E. D., \& Estrs, W. K. Stimulus sampling theorv. San Francisco: Holden-Day, 1967.

Pfautz, P. L., \& W Agner, A. R. Transient variations in respond ing to Pavlovian conditioned stimuli have implications for the mechanism of "priming." Animal Learning \& Behavior, 1976. 4, 107-112.

Rescorla, R. A., \& Wagner, A.R. A theory of Pavlovian conditioning: Variations in the effectiveness of reinforcement and nonreinforcement. In A. H. Black \& W. F. Prokasy (Eds.), Classical conditioning II: Current research and theory. New York: Appleton-Century-Crofts, 1972.

Riley, D. A., \& Roitblat, H. L. Selective attention and related cognitive processes in pigeons. In $\mathrm{S}$. Hulse, $\mathrm{H}$. Fowler, \& W. Honig (Eds.), Cognitive processes in animal behavior. Hillsdale, N.J: Erlbaum, 1978.

Roserts, W. A. Short term memory in the pigeon: Effects of repetition and spacing. Journal of Experimental Psychology, $1972,94,74-83$

Roberts, W. A., \& Grant, D. S. Short term memory in the pigeon with presentation time precisely controlled. Learning and Motivation, 1974, 5, 393-408.

Roberts, W. A., \& Grant, D. S. Studies of short-term memory in the pigeon using the delayed matching to sample procedure. In D. L. Medin, W. A. Roberts, \& R. T. Davis (Eds.), Processes of animal memory. Hillsdale, N.J: Erlbaum, 1976.

Roberts, W. A., \& Grant, D. S. Interaction of sample and comparison stimuli in delayed matching to sample with the pigeon. Journal of Experimental Psychology: Animal Behavior Processes, 1978, 4, 68-82

Rock, 1 . The role of repetition in associative learning. American Journal of Psychology, 1957, 70, 186-193.

RoDewal.D, H. K. All-or-none acquisition in matching-to-sample and a test of two models. Journal of the Experimental Analysis of Behavior, 1974, 22, 53-60.

RoItBlat, H. L. Encoding processes in pigeon short-term memory. Unpublished doctoral dissertation, University of California, Berkeley, 1978.

Theios, J., \& Brelsford, J. W., JR. A Markov model for classical conditioning: Application to eyeblink conditioning in rabbits. Psychological Review, 1966, 73, 393-408

WAGNer, A. R. Priming in STM: An information processing mechanism for self generated or retrieval generated depression in performance. In T. J. Tighe \& R. N. Leaton (Eds.), Habituation: Perspectives from child development, animal behavior and neuropsychology. Hillsdale, N.J: Erlbaum, 1976.

Wickens, T. D., \& GReitzer, F. L. Second responses in pairedassociate learning. Journal of Mathematical Psvchology, 1975 , $12,225-261$

Winograd, E. Some issues relating animal memory to human memory. In W. K. Honig \& P. H. R. James (Eds.), Animal memory. New York: Academic Press, 1971

Worsham, R. W. Temporal discrimination factors in the delayed matching-to-sample task in monkeys. Animal Learning \& Behavior, 1975, 3, 93-97.

Wright, A. A., \& Cumming, W. A. Color naming functions for the pigeon. Journal of the Experimental Analvsis of Behavior. $1971,15,7.17$

(Received for publication March 9. 1979; revision accepted February 12, 1980.) 